\title{
Automatic Removal of Mechanical Fixations from CT Imagery with Particle Swarm Optimisation
}

\author{
Mohammad Hashem Ryalat, Stephen Laycock, and Mark Fisher \\ University of East Anglia, Norwich Research Park, Norwich, NR4 7TJ, UK \\ \{M.Ryalat, S. Laycock, Mark.Fisher\}@uea.ac.uk \\ http://www .uea.ac.uk/computing
}

\begin{abstract}
Fixation devices are used in radiotherapy treatment of head and neck cancers to ensure successive treatment fractions are accurately targeted. Typical fixations usually take the form of a custom made mask that is clamped to the treatment couch and these are evident in many CT data sets as radiotherapy treatment is normally planned with the mask in place. But the fixations can make planning more difficult for certain tumor sites and are often unwanted by third parties wishing to reuse the data. Manually editing the CT images to remove the fixations is time consuming and error prone. This paper presents a fast and automatic approach that removes artifacts due to fixations in CT images without affecting pixel values representing tissue. The algorithm uses particle swarm optimisation to speed up the execution time and presents results from five CT data sets that show it achieves an average specificity of $92.01 \%$ and sensitivity of $99.39 \%$.
\end{abstract}

Keywords: Immobilization Mask, CT Images, Head and Neck Cancer.

\section{Introduction}

Head and Neck Cancer (HNC) refers to a group of different malignant tumors that develop in or around the throat, larynx, nose, sinuses, and mouth [1]. Staging of the cancer may be determined by medical imaging, biopsy and blood tests [2]. HNC is the eighth most common cancer in the UK (2014), accounting for $3 \%$ of all new cases [3]. Figures published from the United States estimate that 61,760 people developed head and neck cancer in 2015 [4].

A course of Radiotherapy Treatment (RT) typically forms a component in the prescribed treatment of $\mathrm{HNC}$ and is delivered in fractions over several weeks. Fixation masks (Figure 1) are employed [5] to ensure the patient can be consistently positioned for each dose fraction. CT data used for planning HNC radiotherapy treatment contain artifacts due to the mask (Figure 6a) which in some cases can make planning more difficult and can be troublesome for third parties wishing to reuse the data. This paper proposes an automatic approach to removing them. 


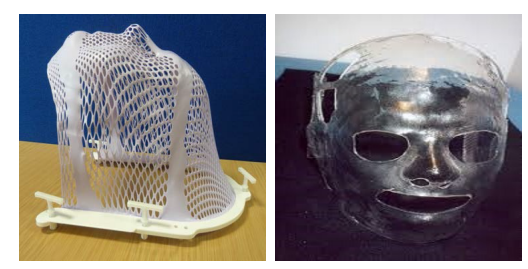

Fig. 1. Immobilization masks (left) Thermoplastic (right) Polyethylene.

Since Head and Neck CT data are usually acquired prior to radiotherapy treatment many publicly available $\mathrm{CT}$ data sets are from patients fitted with immobilization masks or other fixation devices. Segmentation of anatomical structures such as brain, lateral ventricles and skull is required prior to building and rendering 3-D models of these data but artifacts within the CT due to the immobilization mask makes the task more complicated. The removal of artifacts by manually editing individual CT image slices is time consuming and error prone. This is particularly problematic in the regions where the mask contacts the skin [6] [7]. Therefore a robust approach to automatically remove the masks from the CT slices represents an appreciable saving in time.

There are numerous studies related to the segmentation and identification of the head/intra-cranial in the CT images [8-11] but in our knowledge, this study is the first to present a fully automatic approach for removing CT image artifacts due the fixation mask. Our algorithm employs an extension of Otsu's method, which classifies pixels as belonging to one of many classes using multilevel thresholding [5]. Exhaustive search for multiple thresholds requires the evaluation of $(n+1)(D-n+2)^{\mathrm{n}}$ combinations of thresholds, where $n$ represents the number of thresholds and $D$ represents the absolute difference between the maximum and minimum image pixel value. Since pixel intensities in Dicom images are represented by signed integers the search can be very time consuming. To address this we test three optimisation techniques: Particle Swarm Optimisation (PSO)[12], Darwinian Particle Swarm Optimisation (DPSO)[13] and Fractional Darwinian Particle Swarm Optimisation (FDPSO) [14]

The remainder of this paper is organized as follows. Section 2 presents the proposed approach and section 3 presents the data sets used for evaluation and the experiments that were performed. Section 4 presents the results and finally, section 5 draws conclusions.

\section{The Proposed Approach}

The basic steps of the proposed approach are presented in Figure 2. The algorithm applies FDPSO 'slice-by-slice' to segment the image to six different classes under Otsu's criterion [15]. Section 3 explains that we found segmenting the image into six different classes empirically, since in all our experiments this numberclusters all or most of the pixels belonging to the mask as one class. Algorithm 1 further refines the output of the FODPSO segmentation by employing a 
heuristic search for pixels in the labelled image that represent the immobilization mask and background.

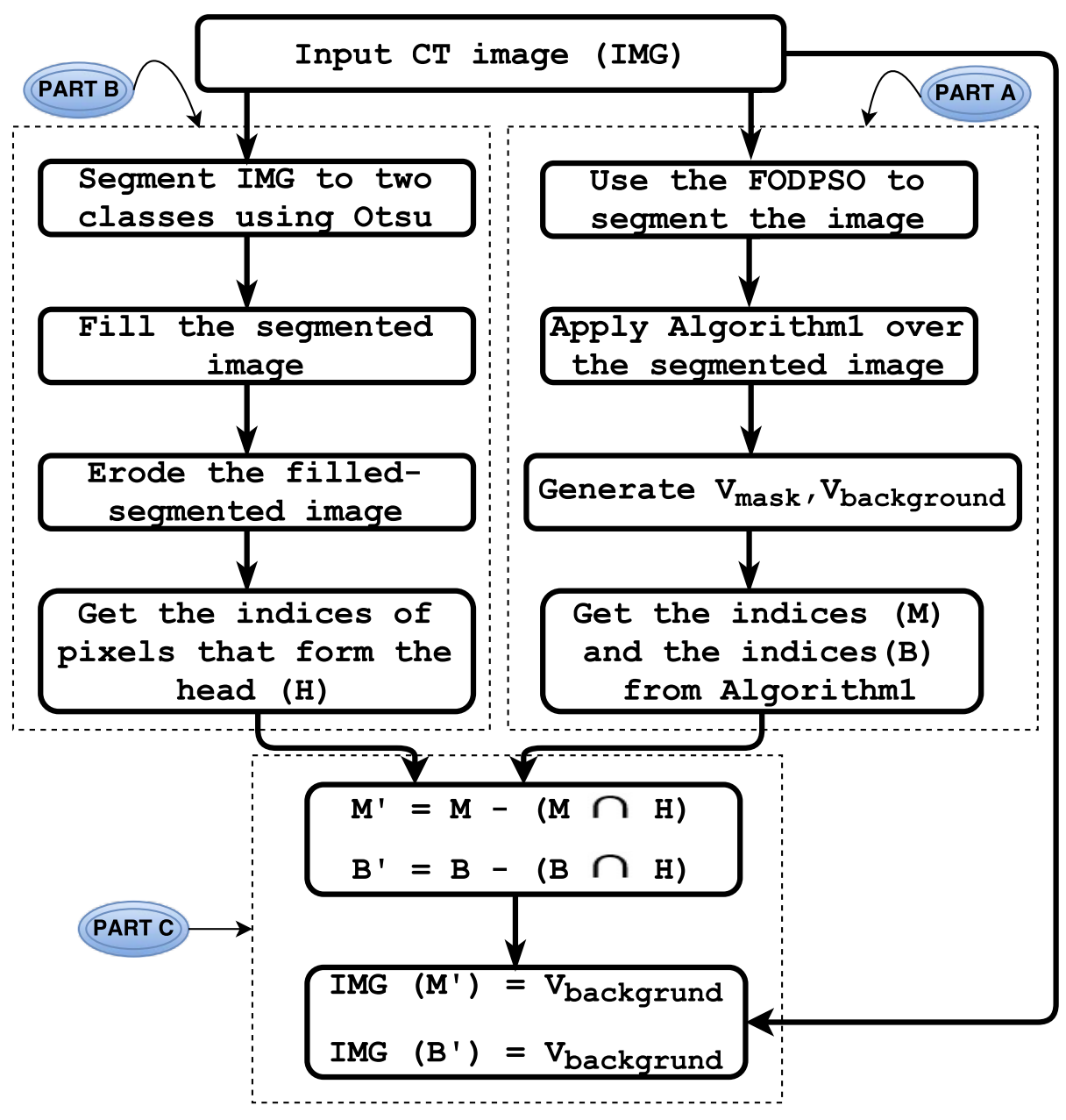

Fig. 2. Overview of the proposed approach.

We assume the top middle pixel represents the image background (air) and search the labeled image until we find a different pixel value (e.g. identified by the red square in Figure 3). This pixel is assumed to belong to the class labelled mask. Using these pixel labels we identify sets of pixels $\{M\}$ and $\{B\}$ that represent the mask and background respectively.

Figure 4 illustrates that $\{M\}$ and $\{B\}$ sometimes include erroneous pixels because the FODPSO segmentation process groups these as one cluster. The sets $\{M\}$ and $\{B\}$ contain pixels that are misclassified because the FDPSO algorithm 

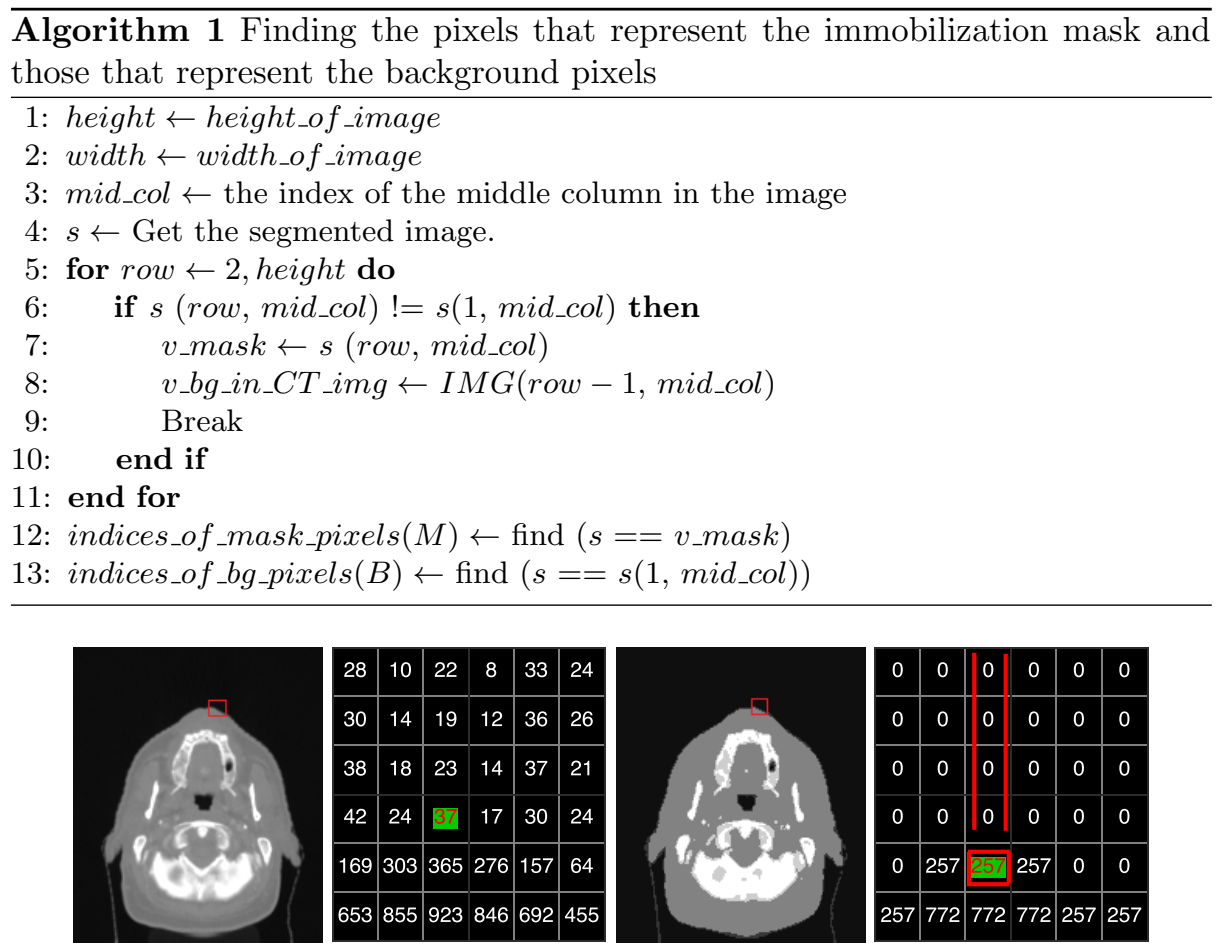

Fig. 3. A CT image in DICOM format and the same image after segmentation using the FODPSO algorithm along with their pixel region tool.

only uses intensity to cluster pixels. In our experiments, illustrated in Figure 4 we found that the misclassified pixels are always located inside the skull. We correct this problem by recovering the coordinates of those pixels located within the skull and excluding these from $\{M\}$ by a sequence of operations that split the original CT image to two clusters (i.e. foreground(head) and background(air)), once again by using Otsu's method. We then flood-fill holes that may appear inside the skull using the morphological reconstruction operator described in [16]. Subsequently we proceed by performing an erosion [17] over the filled image. The aim of this process is to erode away the boundaries of the skull so areas of foreground pixels shrink in size. This will guarantee that none of the pixels that belong to the mask will be excluded later and the only pixels that will be excluded are those which are exist inside the skull. The index of those pixels which represent the skull are recovered from the eroded image as $\{H\}$. Equations (1) and (2) identify the sets $\left\{M^{\prime}\right\}$ and $\left\{B^{\prime}\right\}$ that exclude those pixels within $\{M\}$ and $\{B\}$ that are also with the skull $\{H\}$.

$$
\begin{gathered}
M^{\prime}=M-(M \cap H) \\
B^{\prime}=B-(B \cap H)
\end{gathered}
$$



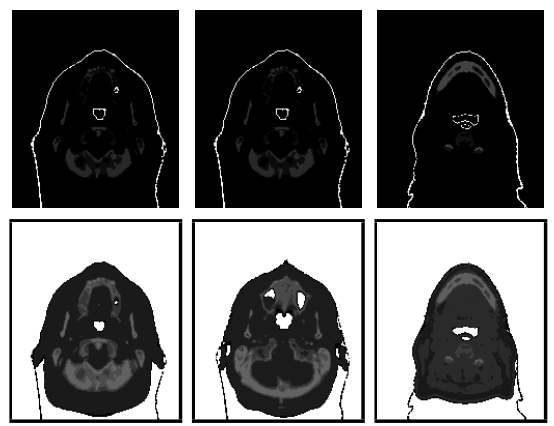

Fig. 4. Examples of pixels mislabeled by FDPSO. Upper row: pixels mislabeled as $\{M\}$; Lower row: pixels mislabeled as $\{B\}$.

\section{Experimental Work}

\section{Data set}

Five CT data sets from anonymized patients have been used in this study. The first three data sets $(512 \times 512 \times 155,512 \times 512 \times 146,512 \times 512 \times 151$, helical, pixelspacing $1.367 \times 1.367 \mathrm{~mm}$, slice-thickness $2.5 \mathrm{~mm}$ ) were acquired at St James's University Hospital NHS Foundation Trust, Leeds, UK and the other two data sets $(512 \times 512 \times 130,512 \times 512 \times 156$, pixel-spacing $1.08 \times 1.08 \mathrm{~mm} 0.98 \times 0.98 \mathrm{~mm}$, slice-thickness $3.14 \mathrm{~mm}$ ) were downloaded from the Cancer Imaging Archive (TCIA)/Head-Neck-Cetuximab [18][19].

\section{Experiments}

The accuracy of the PSO, DPSO and FODPSO is evaluated by measuring the fitness (i.e. inter-class variance) for each algorithm and comparing the outputs with the brute-force (BF) method. Table 1 shows the average fitness values generated by the PSO, DPSO and FODPSO algorithms compared with that generated by the BF method. It is clear from Table 1 that the fitness value produced by the FODPSO algorithm is the same or a slightly less than that of the BF method. This indicates that applying the FODPSO algorithm for segmentation leads to a very high accurate outcomes.

Table 2 displays the average CPU processing time that PSO, DPSO, FODPSO and $\mathrm{BF}$ methods need to segment all image slices in each data set. The table confirms that the FODPSO algorithm is always slightly faster than the DPSO algorithm and the DPSO algorithm is significantly faster than the PSO algorithm. It is worth noting that the speed of BF search is similar to, but less than the speed of FODPSO when the number of thresholds equals one. But as the number of thresholds increases, the difference between the speed of BF and the speed of the other three optimisation algorithms becomes significant. In our case (i.e. removing artifacts due to immobilization masks) we are interested in the 
Table 1. Average fitness values of Brute-Force, PSO, DPSO and FODPSO algorithms for different number of thresholds over different five data sets

\begin{tabular}{llllll}
\hline Dataset & Thr. & BF & PSO & DPSO & FODPSO \\
\hline Dataset\#1 & 1 & 3269.09 & $\mathbf{3 2 6 9 . 0 9}$ & $\mathbf{3 2 6 9 . 0 9}$ & $\mathbf{3 2 6 9 . 0 9}$ \\
(155 images) & 2 & 3773.38 & 3773.37 & $\mathbf{3 7 7 3 . 3 8}$ & $\mathbf{3 7 7 3 . 3 8}$ \\
& 3 & 3829.39 & 3829.36 & $\mathbf{3 8 2 9 . 3 8}$ & $\mathbf{3 8 2 9 . 3 8}$ \\
& 4 & 3855.43 & 3854.92 & 3855.25 & $\mathbf{3 8 5 5 . 3 9}$ \\
& 5 & 3871.88 & 3871.81 & 3871.72 & $\mathbf{3 8 7 1 . 8 2}$ \\
\hline Dataset\#2 & 1 & 3488.54 & 3488.49 & $\mathbf{3 4 8 8 . 5 4}$ & $\mathbf{3 4 8 8 . 5 4}$ \\
(146 images) & 2 & 4067.33 & 4067.21 & $\mathbf{4 0 6 7 . 3 0}$ & $\mathbf{4 0 6 7 . 3 0}$ \\
& 3 & 4142.05 & 4141.87 & 4141.91 & $\mathbf{4 1 4 2 . 0 2}$ \\
& 4 & 4178.69 & 4178.11 & $\mathbf{4 1 7 8 . 6 7}$ & $\mathbf{4 1 7 8 . 6 7}$ \\
& 5 & 4197.88 & 4197.09 & 4197.09 & $\mathbf{4 1 9 7 . 8 6}$ \\
\hline Dataset\#3 & 1 & 2374.66 & 2374.39 & 2374.54 & $\mathbf{2 3 7 4 . 5 4}$ \\
(151 images) & 2 & 2635.29 & 2634.50 & 2634.88 & $\mathbf{2 6 3 5 . 2 6}$ \\
& 3 & 2657.87 & 2654.98 & 2655.29 & $\mathbf{2 6 5 7 . 8 4}$ \\
& 4 & 2679.31 & 2677.82 & 2679.19 & $\mathbf{2 6 7 9 . 2 7}$ \\
& 5 & 2688.69 & 2685.58 & 2686.44 & $\mathbf{2 6 8 8 . 6 4}$ \\
\hline Dataset\#4 & 1 & 3749.77 & $\mathbf{3 7 4 9 . 7 7}$ & $\mathbf{3 7 4 9 . 7 7}$ & $\mathbf{3 7 4 9 . 7 7}$ \\
(130 images) & 2 & 4264.51 & 4264.01 & 4264.32 & $\mathbf{4 2 6 4 . 5 1}$ \\
& 3 & 4363.87 & 4363.12 & 4363.72 & $\mathbf{4 3 6 3 . 8 6}$ \\
& 4 & 4418.97 & 4417.99 & $\mathbf{4 4 1 8 . 9 6}$ & $\mathbf{4 4 1 8 . 9 6}$ \\
& 5 & 4435.87 & 4431.89 & 4435.82 & $\mathbf{4 4 3 5 . 8 5}$ \\
\hline Dataset\#5 & 1 & 2870.42 & 2870.18 & $\mathbf{2 8 7 0 . 4 2}$ & $\mathbf{2 8 7 0 . 4 2}$ \\
(156 images) & 2 & 3386.32 & 3385.83 & 3386.12 & $\mathbf{3 3 8 6 . 3 2}$ \\
& 3 & 3462.11 & 3460.76 & 3460.76 & $\mathbf{3 4 6 2 . 1 0}$ \\
& 4 & 3516.13 & 3515.73 & 3516.08 & $\mathbf{3 5 1 6 . 1 1}$ \\
& 5 & 3535.57 & 3533.12 & 3534.84 & $\mathbf{3 5 3 5 . 5 3}$ \\
\hline
\end{tabular}

case when the number of thresholds equals 5 , and then the use of the FODPSO algorithm will make a significant enhancement in terms of speed. The fractional coefficient used in FODPSO allows the convergence rate of the algorithm to be controlled and this explains why FODPSO outperforms the DPSO algorithm.

The standard deviation was used as an evaluation of stability. Table 3 shows that FODPSO produces the most stable results when compared to the PSO and DPSO, and the standard deviation increases as the number of thresholds increase in most cases. Typical results of segmentation using the FODPSO algorithm over one sample image using different number of thresholds is shown in Figure 5.

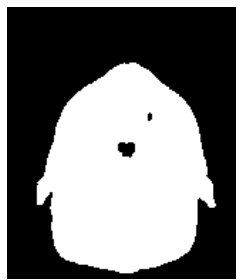

1 threshold

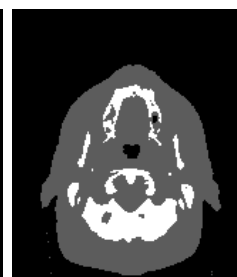

2 thresholds

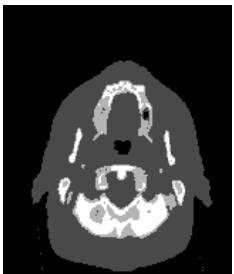

3 thresholds

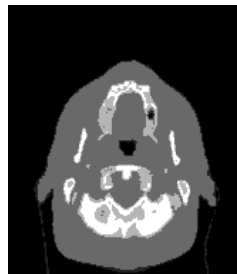

4 thresholds

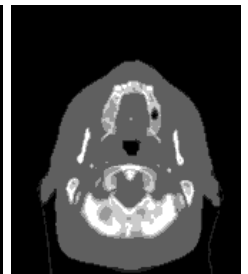

5 thresholds

Fig. 5. Applying FODPSO using different number of thresholds.

Figure 6(a) displays an example of one of the CT slices from the first data set. A previous study [5] evaluated the use of Particle Swarm Optimisation for 
Table 2. Average execution time (in sec) of the Brute-Force, PSO, DPSO and FODPSO algorithms for different number of thresholds over different five data sets

\begin{tabular}{llllll}
\hline Dataset & T.holds & Brute-Force & PSO & DPSO & FODPSO \\
\hline Dataset\#1 & 1 & 10.86 & 33.81 & 12.15 & $\mathbf{9 . 7 2}$ \\
& 2 & 235.52 & 73.12 & 58.38 & $\mathbf{5 6 . 7 5}$ \\
& 3 & 25951 & 90.85 & 74.90 & $\mathbf{7 2 . 5 3}$ \\
& 4 & 2408545 & 111.01 & 90.04 & $\mathbf{8 6 . 8 2}$ \\
\hline Dataset\#2 & 5 & $>1$ week & 131.06 & 103.25 & $\mathbf{9 8 . 0 3}$ \\
& 2 & 7.28 & 20.95 & 8.37 & $\mathbf{6 . 9 6}$ \\
& 3 & 24429 & 65.56 & 53.39 & $\mathbf{5 0 . 2 7}$ \\
& 4 & 1709952 & 84.90 & 71.50 & $\mathbf{6 7 . 2 0}$ \\
& 5 & $>104.41$ & 83.91 & $\mathbf{7 9 . 0 8}$ \\
\hline Dataset\#3 & 1 & 7.25 & 123.61 & 97.84 & $\mathbf{9 3 . 4 2}$ \\
& 2 & 232.58 & 20.20 & 7.03 & $\mathbf{6 . 2 0}$ \\
& 3 & 25118 & 69.35 & 56.92 & $\mathbf{5 4 . 8 5}$ \\
& 4 & 1809433 & 88.02 & 70.82 & $\mathbf{6 6 . 5 4}$ \\
& 5 & $>1$ week & 127.73 & 86.51 & $\mathbf{8 2 . 3 6}$ \\
\hline Dataset\#4 & 1 & 8.06 & 16.15 & 8.51 & $\mathbf{7 . 9 7}$ \\
& 2 & 195.84 & 58.54 & 47.82 & $\mathbf{4 6 . 6 9}$ \\
& 3 & 21717 & 75.35 & 61.32 & $\mathbf{6 0 . 8 0}$ \\
& 4 & 1908530 & 92.85 & 76.12 & $\mathbf{7 2 . 9 1}$ \\
& 5 & $>1$ week & 109.67 & 86.73 & $\mathbf{8 4 . 1 6}$ \\
\hline Dataset\#5 & 1 & 9.26 & 49.02 & 8.75 & $\mathbf{8 . 0 9}$ \\
& 2 & 238.19 & 70.40 & 57.81 & $\mathbf{5 7 . 5 9}$ \\
& 3 & 26148 & 90.99 & 76.13 & $\mathbf{7 2 . 1 2}$ \\
& 4 & 2173860 & 111.82 & 90.04 & $\mathbf{8 4 . 2 9}$ \\
& 5 & $>1$ week & 132.30 & 104.59 & $\mathbf{9 7 . 2 1}$ \\
\hline
\end{tabular}

medical image segmentation and demonstrated the the FODPSO algorithm delivered high accuracy, stability and speed. We found that for our application, segmenting the image to six different classes tends to lead to better results as this number classifies all or most of the pixels belonging to the mask as one class. The FODPSO algorithm delivers significant benefits in terms of execution speed over the BF approach (i.e. exhaustive search) which takes a very long time when the number of clusters equals six. In Section 4 we compare the FODPSO algorithm with other techniques by tabulating the run time needed to segment a typical HNC CT data set. Figure 6(b) displays the image after it was segmented to six different clusters using the FODPSO algorithm.

In Figure 6(c-e) we present the output that is generated by part-B of the proposed approach. The image was firstly segmented to two classes (foreground and background) using Otsu's method. It was then filled automatically and eroded as it displayed in 6(e). Part-A and part-B of the proposed approach produced three data structures of indices $(M, B$, and $H)$ and those indices were used to form the final output image which is displayed in Figure 6(f). Finally, Figure 7 illustrates randomly-selected input images and their outputs after applying the approach. 
Table 3. Standard deviation of fitness for PSO, DPSO and FODPSO after running each algorithm 15 times over different five data sets.

\begin{tabular}{lllll}
\hline Dataset & T.holds & PSO & DPSO & FODPSO \\
\hline Dataset\#1 & 1 & $\mathbf{0}$ & $\mathbf{0}$ & $\mathbf{0}$ \\
& 2 & 0.0001 & $\mathbf{0}$ & $\mathbf{0}$ \\
& 3 & 0.0036 & 0.0003 & $\mathbf{0 . 0 0 0 2}$ \\
& 4 & 1.2873 & 0.0114 & $\mathbf{0 . 0 1 0 5}$ \\
& 5 & 0.0206 & 1.2195 & $\mathbf{0 . 0 1 9 0}$ \\
\hline Dataset\#2 & 1 & 0.0001 & $\mathbf{0}$ & $\mathbf{0}$ \\
& 2 & 0.0009 & 0.0002 & $\mathbf{0 . 0 0 0 1}$ \\
& 3 & 0.0021 & 0.0005 & $\mathbf{0 . 0 0 0 2}$ \\
& 4 & 0.0122 & 0.0120 & $\mathbf{0 . 0 1 1 3}$ \\
& 5 & 0.0787 & 0.0342 & $\mathbf{0 . 0 3 4 1}$ \\
\hline Dataset\#3 & 1 & 0.0023 & 0.0001 & $\mathbf{0}$ \\
& 2 & 0.0082 & 0.0009 & $\mathbf{0 . 0 0 0 1}$ \\
& 3 & 0.0810 & 0.0569 & $\mathbf{0 . 0 0 1 5}$ \\
& 4 & 0.0254 & 0.0143 & $\mathbf{0 . 0 0 6 1}$ \\
\hline Dataset\#4 & 5 & 0.5932 & 0.5437 & $\mathbf{0 . 2 9 0 3}$ \\
& 1 & 0.0011 & 0.0002 & $\mathbf{0}$ \\
& 2 & 0.0008 & 0.0002 & $\mathbf{0 . 0 0 0 1}$ \\
& 3 & 0.0110 & 0.0089 & $\mathbf{0 . 0 0 7 3}$ \\
& 4 & 0.0196 & 0.0159 & $\mathbf{0 . 0 1 6 1}$ \\
& 5 & 1.7163 & 0.0938 & $\mathbf{0 . 0 8 8 4}$ \\
\hline Dataset\#5 & 1 & 0.0005 & 0.0003 & $\mathbf{0 . 0 0 0 1}$ \\
& 2 & 0.0012 & 0.0004 & $\mathbf{0 . 0 0 0 1}$ \\
& 3 & 0.0082 & 0.0027 & $\mathbf{0 . 0 0 1 1}$ \\
& 4 & 0.0243 & 0.0199 & $\mathbf{0 . 0 1 9 0}$ \\
& 5 & 1.3081 & 0.0373 & $\mathbf{0 . 0 3 6 1}$ \\
\hline
\end{tabular}

\section{Results, Validation and Discussion}

\section{The Performance of the Approach}

We used the Sensitivity and the Specificity to evaluate the proposed approach as both of them are statistical measures of the performance of a binary classification test. We have identified the True Positive (TP), False Positive (FP), True Negative (TN) and False Negative(FN) in this context as:

- TP: \# of mask pixels correctly identified as mask.

- FP: \# of not-a-mask pixels incorrectly identified as mask.

- TN: \# of not-a-mask pixels correctly identified as not-a-mask.

- FN: \# of mask pixels incorrectly identified as not-a-mask.

The pixels that represent the immobilisation mask were identified by an expert in $25 \mathrm{CT}$ images (5 randomly-selected from each dataset) and compared to the number of pixels identified by the proposed approach. Table 4 displays the average values, rounded to the whole number, of TP, FP, TN and FN for each dataset and Table 5 displays the sensitivity, also called the true positive rate (TPR), specificity (SPC) and the Number-Of-Observations (NOO) for each dataset.

As is shown in Table 5 the average value of the sensitivity (TPR) is $92.01 \%$ which indicates to the proportion of positives that are correctly identified (i.e. the percentage of mask pixels which are correctly identified by the proposed approach as mask pixels) and the average value of the specificity (SPC) is $99.39 \%$ 

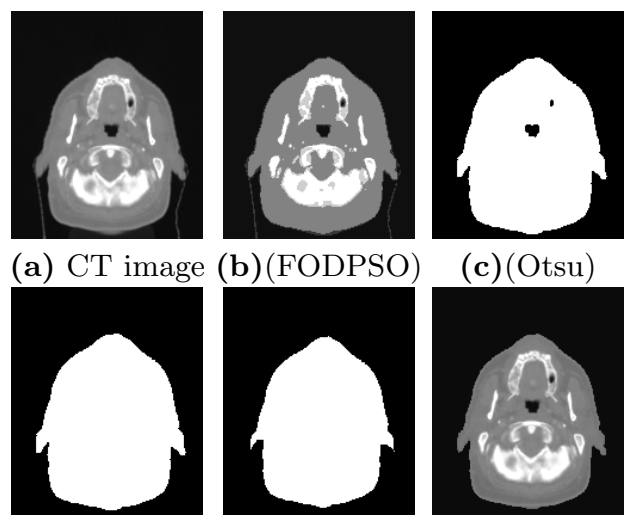

(b) FODPSO

(c) (Otsu)

(d) Filled

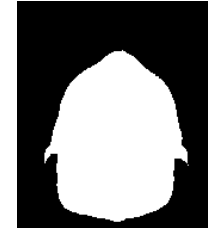

(e) Eroded

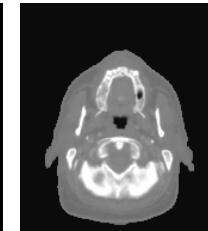

(f) Output

Fig. 6. An example of a CT slice from the first dataset.

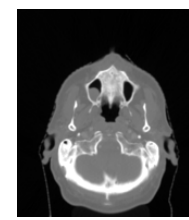

(a) In_D2

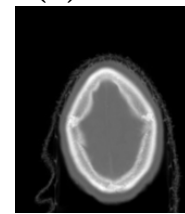

(e) In_D4

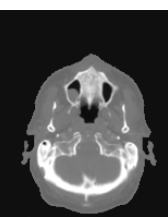

(b) Out_D2

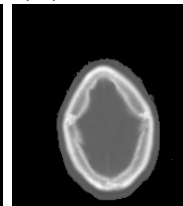

(f) out_D4

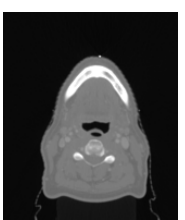

(c) In_D3

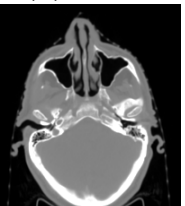

(g) In_D5

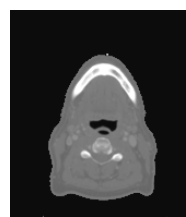

(d) Out_D3

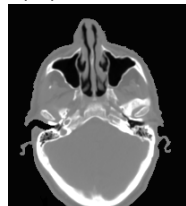

(h) Out_D5

Fig. 7. One CT slice example from each dataset (Input Output).

which points to the proportion of negatives that are correctly identified (i.e. the percentage of not-a-mask pixels who are correctly identified as not-a-mask pixels). The heading 'NOO' in the table indicates to the number of observations which is equivalent to the number of pixels in each image.

\section{Handling Exceptions}

We applied our approach over five different data sets (total $=738$ images) and noticed that the approach did not work on 13 images of them. This accounts for two reasons (1) some CT images include a noise in the middle column on the top of the mask itself, as displayed in Figure 8-left (2) some CT images have disconnected representation of the mask pixels, as displayed in Figure 8middle. We handled the first exception by applying the median filter over the background area in order to remove the noise from the background area, and we 
Table 4. The average values of TP, FP, TN, and FN for each dataset

\begin{tabular}{lllll}
\hline Dataset & TP & TN & FP & FN \\
\hline Dataset\#1 & 389 & 30,239 & 100 & 23 \\
Dataset\#2 & 403 & 30,152 & 154 & 42 \\
Dataset\#3 & 429 & 30,199 & 93 & 30 \\
Dataset\#4 & 1714 & 29,060 & 465 & 203 \\
Dataset\#5 & 841 & 45,371 & 148 & 71 \\
\hline
\end{tabular}

Table 5. The values of TPR, SPC, and NOO for each dataset

\begin{tabular}{llll}
\hline Dataset & TPR & SPC & NOO \\
\hline Dataset\#1 & 0.9441 & 0.9967 & 30751 \\
Dataset\#2 & 0.9056 & 0.9949 & 30751 \\
Dataset\#3 & 0.9346 & 0.9969 & 30751 \\
Dataset\#4 & 0.8941 & 0.9842 & 31442 \\
Dataset\#5 & 0.9221 & 0.9967 & 46431 \\
Average & $\mathbf{0 . 9 2 0 1}$ & $\mathbf{0 . 9 9 3 9}$ & 34,025 \\
\hline
\end{tabular}

handled the second exception by changing the seeking mechanism in Algorithm 1 by searching the segmented image horizontally and vertically from different five start points as displayed in Figure 8-right.
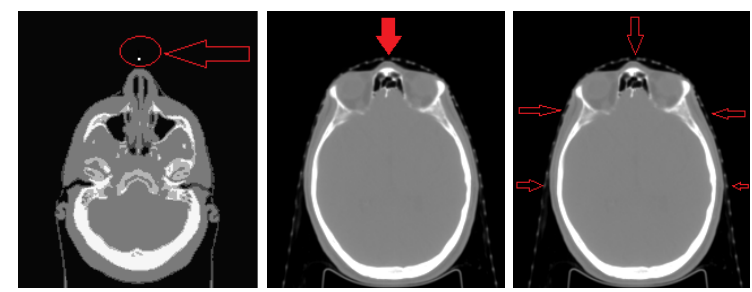

Fig. 8. (Left) Example of a CT image includes a noise in the middle column (Middle) A CT image has a disconnected representation of the mask pixels (Right) Defining new start points to seek horizontally.

\section{Conclusion}

This paper presented an automatic approach for segmenting immobilization masks in Head-and-Neck CT data sets. The approach identifies the pixels that belong to the immobilization mask and replaces their intensity value with that of air, thereby eliminating the mask from the output image. Five different data sets were tested to evaluate the accuracy of the approach. Sensitivity and specificity were used as statistical measures of the performance of the approach in this study. The evaluation indicates that the proposed approach is robust and of 
practical use. Some enhancements to speed up the process using Particle Swarm Optimisation were also presented and tested in the paper.

\section{Acknowledgement}

We would like to thank Prof. Susan Short and colleagues at St James's University Hospital NHS Foundation Trust for their help and for providing some of the data used in this study.

\section{References}

1. Head and Neck Cancer - Overview. http://www.cancer.net/cancer-types/ head-and-neck-cancer/overview. Accessed: 27-11-2016.

2. Head and Neck Cancers. https://www.cancer.gov/types/head-and-neck/ head-neck-fact-sheet. Accessed: 27-11-2016.

3. The Office for National Statistics Report. https://www.ons.gov.uk/. Accessed: 27-11-2016.

4. Head and Neck Cancer: Statistics. http://www.cancer.net/cancer-types/ head-and-neck-cancer/statistics. Accessed: 27-11-2016.

5. Mohammad Hashem Ryalat, Daniel Emmens, Mark Hulse, Duncan Bell, Zainab Al-Rahamneh, Stephen Laycock, and Mark Fisher. Evaluation of particle swarm optimisation for medical image segmentation. In International Conference on Systems Science, pages 61-72. Springer, 2016.

6. Mark Fisher, Christopher Applegate, Mohammad Ryalat, Stephen Laycock, Mark Hulse, Daniel Emmens, Duncan Bell, et al. Evaluation of 3-D printed immobilisation shells for head and neck imrt. Open Journal of Radiology, 4(04):322, 2014.

7. SD Laycock, M Hulse, CD Scrase, MD Tam, S Isherwood, DB Mortimore, D Emmens, J Patman, SC Short, and GD Bell. Towards the production of radiotherapy treatment shells on 3d printers using data derived from dicom CT and MRI: preclinical feasibility studies. Journal of Radiotherapy in Practice, 14(01):92-98, 2015.

8. Kaiping Wei, Bin He, Tao Zhang, and Xianjun Shen. A novel method for segmentation of CT head images. In 2007 1st International Conference on Bioinformatics and Biomedical Engineering, pages 717-720. IEEE, 2007.

9. Wenan Chen, Rebecca Smith, Soo-Yeon Ji, and Kayvan Najarian. Automated segmentation of lateral ventricles in brain CT images. In Bioinformatics and Biomeidcine Workshops, 2008. BIBMW 2008. IEEE International Conference on, pages 48-55. IEEE, 2008.

10. Xiaohua Qian, Jiahui Wang, Shuxu Guo, and Qiang Li. An active contour model for medical image segmentation with application to brain CT image. Medical physics, 40(2):021911, 2013.

11. W Mimi Diyana W Zaki, M Faizal A Fauzi, R Besar, and WSH Munirah W Ahmad. Qualitative and quantitative comparisons of haemorrhage intracranial segmentation in CT brain images. In TENCON 2011-2011 IEEE Region 10 Conference, pages 369-373. IEEE, 2011.

12. Russ C Eberhart, James Kennedy, et al. A new optimizer using particle swarm theory. In Proceedings of the sixth international symposium on micro machine and human science, volume 1, pages 39-43. New York, NY, 1995. 
13. Jason Tillett, T Rao, Ferat Sahin, and Raghuveer Rao. Darwinian particle swarm optimization. In B Prasad, editor, Proceedings of the 2nd Indian International Conference on Artificial Intelligence (IICAI-05), pages 1474-1487, 2005.

14. Micael S Couceiro, Rui P Rocha, NM Fonseca Ferreira, and JA Tenreiro Machado. Introducing the fractional-order darwinian pso. Signal, Image and Video Processing, 6(3):343-350, 2012.

15. Nobuyuki Otsu. A threshold selection method from gray-level histograms. Automatica, 11(285-296):23-27, 1975.

16. Soille Pierre. Morphological image analysis: Principles and applications, 1999.

17. Rafael C Gonzalez and Richard E Woods. Image processing. Digital image processing, 2, 2007.

18. Kenneth Clark, Bruce Vendt, Kirk Smith, John Freymann, Justin Kirby, Paul Koppel, Stephen Moore, Stanley Phillips, David Maffitt, Michael Pringle, et al. The cancer imaging archive (TCIA): maintaining and operating a public information repository. Journal of digital imaging, 26(6):1045-1057, 2013.

19. The Cancer Imaging Archive. http://doi.org/10.7937/K9/TCIA.2015. 7AKGJUPZ/. Bosch, Walter R. and Straube, William L. and Matthews, John W. and Purdy, James A. Data From Head Neck Cetuximab (2015). 